\title{
Aging, short-term memory, and frontal dysfunction
}

\author{
ALAN J. PARKIN and BRENDA M. WALTER \\ University of Sussex, Brighton, England
}

\begin{abstract}
In this paper, we examine the performance of young and old subjects on the Brown-Peterson (BP) task and on a task of recognition memory. Age-related deficits were found on both tasks. For old subjects, BP performance was positively related to both recognition accuracy and intactness of frontal lobe function, as measured by word fluency and the Wisconsin Card Sorting Test (WCST). Recognition accuracy did not correlate with either frontal measure, and no correlations of theoretical significance were found in the younger group. The data are interpreted as showing that age-related differences in BP performance reflect two factors-a deficit in initial acquisition, and a retrieval deficit stemming from the frontal atrophy known to be associated with normal aging.
\end{abstract}

The Brown-Peterson (BP) task (Brown, 1959; Peterson \& Peterson, 1959) is a widely used measure of short-term memory function. ${ }^{1}$ Briefly, the task involves the presentation of target stimuli, usually words, followed by a variable retention interval filled with distracting activity, after which the subject attempts to recall the target stimuli. Examination of performance at each distractor interval then provides a measure of an individual's rate of forgetting.

Comparisons of young and old subjects on the BP task have shown that young subjects perform better (see Kausler, 1982, for a review of these studies). This age difference cannot, however, be attributed to a faster forgetting rate in older subjects, because the age difference in BP performance has been found to be invariant as a function of retention interval. Instead, the data suggest that the deficit arises from defective acquisition/storage, some impairment in retrieval processes, or both. Our purpose in this paper is to explore further the nature of age-related impairments on the BP task.

In a recent paper, Leng and Parkin (1989) compared BP performance in a group of Korsakoff patients and a group of patients with primary medial temporal lesions. It was found, in line with previous studies, that Korsakoff patients performed the BP task more poorly than did the medial temporal group. Additional analyses indicated that the performance difference between the two patient groups was not attributable to variations in patients' memory abilityas measured through a "composite memory score." Instead, it was found that BP performance correlated significantly with performance on the Wisconsin Card Sorting Test (WCST), a widely used test of frontal lobe dysfunction, and that this correlation was most evident

This research was supported by the U.K. Medical Research Council. The authors acknowledge the help of Nikki Hunkin in carrying out this study. Preparation of this paper was aided by grants from the Erskine Foundation, University of Canterbury, and the Raine Foundation, University of Western Australia, to the first author. Please address all correspondence to A. J. Parkin, Laboratory of Experimental Psychology, University of Sussex, Brighton, East Sussex BN1 9QG, England. in the Korsakoff group. However, dysfunction involving frontal structures was not the sole basis for explaining impaired BP performance, because the medial temporal group, in whom the correlation between frontal dysfunction and BP performance was very weak, still exhibited impaired performance.

Leng and Parkin (1989) suggested that the correlation between frontal dysfunction and BP performance reflected some deficiency at the stage of retrieval. There is no direct evidence for a critical retrieval factor underlying forgetting in the BP task, but evidence from a related paradigm, release from proactive interference (PI), does support this view. Gardiner, Craik, and Birtwhistle (1972) found that release from PI could be induced by the provision of cues, at the point of recall, for target items that did not give release effects when no cues were provided. Leng and Parkin (1989) did not speculate on other factors responsible for poor patient performance, but one reasonable hypothesis is that acquisition and storage factors also were significant. Leng and Parkin's failure to find a correlation between BP performance and their patients' composite memory scores might appear to contradict a defective acquisition/storage explanation. It should be borne in mind, however, that Leng and Parkin's patients were all densely amnesic, and, as such, floor effects may have masked the contribution of this factor to the overall pattern of impaired BP performance.

In the last decade, there has been increasing evidence that the process of normal aging is associated with cortical atrophy, particularly in the frontal cortex. Anatomical and physiological studies (e.g., Gerard \& Weisberg, 1986; Haug, 1983; McEntee \& Crook, 1990; Squire, 1987) have been complemented by behavioral evidence indicating the similarity between normal aging and symptoms associated with focal frontal dysfunction (e.g., Mittenberg, Seidenberg, O'Leary, \& DiGuilio, 1989). Furthermore, it is now established that frontal lobe structures play an important role in memory function. Studies of memory-impaired patients have indicated that frontal 
dysfunction can underlie defective memory for temporal context (e.g., Parkin, Leng, Stanhope, \& Smith, 1988; Schacter, Harbluk, \& McLachlan, 1984; Squire, 1982), poor metamemory (Janowsky, Shimamura, \& Squire, 1989; Parkin, Bell, \& Leng, 1988), and failure to show release from PI (Parkin, Leng, et al., 1988; Squire, 1982).

If the memory loss associated with normal aging is, in any way, related to age-related decline in frontal lobe function, memory deficits revealed in neurological patients with frontal damage might also arise in normal elderly people. Several recent studies have supported this possibility. Craik, Morris, Morris, and Loewen (1990) and Parkin and Walter (1991) have both shown that impaired memory for temporal context in the elderly is linked to frontal dysfunction as measured by psychometric testing, and Moscovitch and Winocur (1983) reported that failure to release from PI can be observed in the institutionalized elderly $-a$ finding that the authors attributed to impaired frontal functioning in this group. Furthermore, the same study showed that environmental cues given at retrieval could induce release effects in elderly subjects that did not occur when the subjects were tested under standard experimental conditions (see also a subsequent study by Winocur, Moscovitch, \& Witherspoon, 1987). This finding strengthens the view that failure to release from PI can arise through faulty retrieval mechanisms, and the association of this failure with frontal dysfunction suggests that the critical retrieval process may be frontally based.

The involvement of frontal lobe dysfunction in agerelated memory impairment, particularly in the releasefrom-PI task, and the indication that BP performance is linked to frontal function, leads to the possibility that the deficit shown by the elderly on the BP task may also be significantly influenced by the status of frontal lobe function. In the following study, the BP performance of normal elderly and young subjects is examined in relation to psychometric tests of frontal lobe function and a measure of recognition memory. Comparisons between BP performance and subjects' performance on these other measures are used to provide an indication of the factors underlying any observed age-related decrements.

\section{METHOD}

\section{Subjects}

All subjects were believed to be in good health for their age at the time of testing. The old group consisted of 20 adults, who, apart from 2 in residential care, lived independent lives in their own homes. The young group comprised 20 adults, drawn from secretarial, administrative, technical, or academic staff, or students at Thames Polytechnic. Subjects were matched on the New Adult Reading Test Full Scale IQ procedure (NART FSIQ-Nelson \& O'Connell, 1978). Older subjects were screened for dementia, using the Cognitive Estimation Test (CET-Shallice \& Evans, 1978) and the Anomalous Sentences Repetition Test (Weeks, 1988). The subjects were also screened for previous neurological illness (e.g., stroke), and any with diet-controlled diabetes were also excluded.
Table 1

General Characteristics of Experimental Groups

\begin{tabular}{lrrrrr}
\hline & \multicolumn{2}{c}{ Old* $^{*}$} & & \multicolumn{2}{c}{ Young $\dagger$} \\
\cline { 2 - 3 } \cline { 5 - 6 } & \multicolumn{1}{c}{$M$} & $S D$ & & $M$ & $S D$ \\
\hline Age (years) & 80.0 & 5.1 & & 33.9 & 6.0 \\
NART FSIQ & 117.6 & 7.2 & & 117.5 & 3.5 \\
CET & 3.2 & 2.4 & & N/A & \\
Anomalous sentences repetition & 4.1 & 4.5 & & N/A & \\
\hline
\end{tabular}

Note-NART FSIQ = National Adult Reading Test Full Scale IQ. $\mathrm{CET}=$ Cognitive Estimation Test, $\mathrm{N} / \mathrm{A}=$ not administered. $* 9$ males, 11 females. +6 males, 14 females.

As a result of these tests, 2 subjects from the initial pool were excluded. Details of the subject groups are shown in Table 1.

\section{Procedure}

The subjects were administered two experimental tasks: BP and recognition memory.

Brown-Peterson task. In the BP procedure, each trial began with the presentation of three words (drawn from the Toronto word pool-F. I. M. Craik, personal communication) on index cards, and the subjects were instructed to try to remember the words. Each word trial was presented for $3 \mathrm{sec}$, after which a three-figure number was presented, and the subjects were required to count backward in threes from that number for the specified retention interval. Two elderly subjects had difficulty with this distractor task and were permitted to subtract in twos. At the end of the retention interval, the subjects were instructed to recall the target items in any order. Five trials were given at retention intervals of 18,30 , and $60 \mathrm{sec}$ in a random sequence. Shorter retention intervals were not examined, because of indications that relationships between BP performance and frontal function only emerge when BP performance is examined over longer retention intervals (Leng \& Parkin, 1989).

Recognition task. The general procedure for this test was that used by Gardiner and Parkin (1990), except that the stimulus material was printed on index cards. Seventy-two target words were arranged in two lists of 36 , which were rotated across the study so that one or the other was presented to each subject. The subjects were shown each word from one of the lists for $2 \mathrm{sec}$ and asked to remember it. After seeing all 36 words, the subjects were occupied for $10 \mathrm{~min}$ in genial conversation (old group) or practice on an embedded figures task (young group). At recognition testing, the subjects were given all 72 words on a single sheet and asked to encircle any word they recognized from the learning list. ${ }^{2}$

Frontal lobe tests. The two frontal lobe tests administered were the modified WCST (Nelson, 1976) and the FAS word fluency (Benton, Hamsher, Varney, \& Spreen, 1983). The two memory tasks were administered on different sessions. Frontal lobe tests were administered at various times. Young subjects were tested individually in a quiet room near their work place; elderly subjects were tested in their own homes.

\section{RESULTS}

\section{Brown-Peterson}

The mean number of items recalled correctly at each retention interval was calculated, and these data are shown in Table 2. A two-way analysis of variance with age and retention interval as fixed factors revealed that the elderly group performed more poorly $[F(1,38)=47.8, p<.0001]$, and that recall declined as the retention interval increased 
$[F(2,76)=9.3, p<.0002]$. The interaction between the fixed factors was not significant $(F<1)$.

\section{Recognition Task}

Recognition accuracy for the young and old was calculated using $d^{\prime}$, and these data are also summarized in Table 2. Comparison of these values indicated a significantly better performance in the younger group $[t(38)=$ $3.63, p<.001]$.

\section{Frontal Lobe Tests}

The data from these tests are summarized in Table 3. Comparison using unrelated $t$ tests indicated that the older subjects performed more poorly on all tests of frontal function (see Table 3).

\section{Correlational Analyses}

The first set of correlations concerned relationships between BP performance, recognition performance, frontal score, age, and IQ. These correlations, which may be found in Table 4, showed that, for the elderly group, BP performance correlated negatively with nonperseverative errors on the WCST, and positively with recognition memory. The correlations between FAS word fluency and total errors on the WCST also approached significance.

Performance on the WCST is known to correlate significantly with age (Haaland, Vranes, Goodwin, \& Garry, 1987), and Miller (1984) has shown that the use of FAS as a measure of frontal dysfunction can be confounded by an additional correlation between FAS and IQ. It was therefore decided to examine the observed correlations between BP performance and frontal measures further, by using second-order partial correlations that removed

Table 2

Number of Words Correctly Recalled, as a Function of Age and Retention Interval, and Recognition Accuracy $\left(d^{\prime}\right)$, as a Function of Age

\begin{tabular}{|c|c|c|c|c|c|c|c|c|}
\hline & \multicolumn{6}{|c|}{ Retention Interval } & \multirow{2}{*}{\multicolumn{2}{|c|}{$d^{\prime}$}} \\
\hline & \multicolumn{2}{|c|}{$18 \mathrm{sec}$} & \multicolumn{2}{|c|}{$30 \mathrm{sec}$} & \multicolumn{2}{|c|}{$60 \mathrm{sec}$} & & \\
\hline & $M$ & $S D$ & $M$ & $S D$ & $M$ & $S D$ & $M$ & $S D$ \\
\hline Young & 13.5 & 0.31 & 13.2 & 0.34 & 11.9 & 0.58 & 2.7 & 0.16 \\
\hline Old & 8.7 & 0.60 & 8.9 & 0.56 & 7.3 & 0.81 & 1.9 & 0.17 \\
\hline
\end{tabular}

Table 3

Comparison of Young and Old Groups on Tests of Frontal Function

\begin{tabular}{lrlrrl}
\hline & \multicolumn{2}{c}{ Young } & & \multicolumn{2}{c}{ Old } \\
\cline { 2 - 3 } \cline { 5 - 6 } & $M$ & \multicolumn{1}{c}{$S E$} & & $M$ & \multicolumn{1}{c}{$S E$} \\
\hline FAS & 58.3 & 2.8 & & 40.0 & 2.6 \\
WCSTc & 6.0 & 0 & & 5.2 & 0.29 \\
WCSTt & 1.7 & 0.45 & & 11.3 & 2.3 \\
WCSTp & 0.3 & 0.13 & & 5.0 & 1.6 \\
WCSTe & 1.4 & 0.36 & & 6.4 & 0.98 \\
\hline
\end{tabular}

Note-FAS $=$ word fluency. WCST $=$ Wisconsin Card Sorting Test. WCSTc $=$ WCST categories achieved. WCSTt $=$ WCST total errors. WCSTp $=$ WCST perseverative errors. WCSTe $=$ WCST nonperseverative errors. $t(38), p<.001$.
Table 4

Correlations Between Brown-Peterson Task Performance (BP), Recognition Performance, and Psychometric Measures

\begin{tabular}{lccccc}
\hline & \multicolumn{2}{c}{ Young } & & \multicolumn{2}{c}{ Old } \\
\cline { 2 - 3 } \cline { 5 - 6 } & BP & Recognition & & BP & Recognition \\
\hline FAS & 0.33 & 0.02 & & 0.34 & 0.01 \\
WCSTt & -0.21 & 0.02 & & -0.35 & 0.01 \\
WCSTp & & & & -0.22 & 0.09 \\
WCSTe & -0.08 & 0.00 & & $-0.47^{*}$ & -0.12 \\
Age & $-0.46 \dagger$ & 0.06 & & 0.15 & 0.09 \\
IQ & 0.23 & $0.48 \dagger$ & & 0.21 & 0.20 \\
Recognition & -0.05 & & & $0.53 \dagger$ & \\
\hline
\end{tabular}

Note-FAS $=$ word fluency. WCST $=$ Wisconsin Card Sorting Test. $\mathrm{BP}=$ total words recalled on BP task. Recognition = recognition accuracy $\left(d^{\prime}\right)$. WCSTt $=$ WCST total errors. WCSTp $=$ WCST perseverative errors. WCSTe $=$ WCST nonperseverative errors. $\quad * p<.05$ (one-tail). $\quad \dagger p<.01$ (one-tail).

Table 5

Old Subjects Only: Results of Second Order Partial Correlations Between Brown-Peterson Task (BP) Performance and Psychometric Measures of Frontal Function

\begin{tabular}{lc}
\hline & BP \\
\hline FAS & $0.47^{*}$ \\
WCSTt & $-0.46^{*}$ \\
WCSTp & -0.35 \\
WCSTe & $-0.50^{*}$ \\
\hline
\end{tabular}

Note-BP $=$ total words recalled on BP task. FAS $=$ word fluency. WCSTt $=$ WCST total errors. WCST $p=$ WCST perseverative errors. WCSTe $=$ WCST nonperseverative errors. $\quad{ }^{*} p<.01$ (one-tail).

the relationship between the frontal measures age and IQ. These data, shown in Table 5, indicate that partialing out age and IQ strengthens the correlations between BP performance and the frontal measures.

\section{DISCUSSION}

The results of this experiment replicated those found by previous authors in showing an age-related decline in BP performance but no interaction between age and retention interval. As noted earlier, the failure to find this interaction argues against a forgetting-rate explanation of age differences in BP performance, because this would predict an increasing effect with age as retention interval increased. Instead, the data indicate that another factor or other factors may account for the observed age differences.

Two possible explanations are revealed by the correlational analyses. First, for the elderly, there is a strong correlation between BP performance and recognition accuracy. If one accepts that recognition is the retention measure least sensitive to variations in retrieval efficiency, the correlation between recognition accuracy and BP performance can be interpreted as showing a positive relationship between BP performance and the processes of acquisition and storage.

The presence of an additional set of correlations between BP performance, FAS performance, and perfor- 
mance on the WCST, and the absence of even marginal correlations with recognition memory, indicates that an additional factor is influencing BP performance that is not affecting recognition accuracy. Maintaining our assumption that recognition is a primary measure of acquisition and storage, one can further speculate that the additional factor reflected in the correlations between BP performance and frontal measures concerns some aspect of retrieval. As mentioned above, there is evidence that a related short-term memory paradigm, release from PI, has a critical retrieval component (Gardiner et al., 1972; Moscovitch \& Winocur, 1983), so it would not be unreasonable to suppose that a similar state of affairs applies to the BP task. Moreover, one should note that deficiencies in the retrieval system are thought to have an important influence on memory impairments arising from focal frontal lesions (e.g., Baddeley, 1987).

A final issue is whether the relation between BP performance and frontal dysfunction reflects frontal involvement per se, or whether it reflects disruption of projections originating in midline diencephalic structures and terminating in the frontal cortex. Although there are no conclusive data on this issue, there are indications that the latter is the more likely interpretation. Parkin, Hunkin, and Rees (1991), using the same version of the BP task as that described here, report BP performance in an amnesic patient with a discrete thalamic infarction. The patient scored 6, 5, and 6 items correct across retention interval-scores comparable to those of the present older group. Parkin, Leng, et al.'s (1988) anterior communicating artery aneurysm case, J.B., also performed very poorly on the BP task even though WCST performance was normal. Case B.Y. (Winocur, Oxbury, Roberts, Agnetti, \& Davis, 1984), who also had a discrete thalamic lesion, performed poorly on the BP task despite normal performance on psychometric tests of frontal functions. Finally, Stuss et al. (1982) observed impaired poor BP performance in schizophrenic patients who had undergone prefrontal leucotomy. Thus, although not conclusive, these findings suggest that impairment on the BP task can be related to some disconnection between frontal and diencephalic structures, rather than purely frontally based.

Data from the younger subjects offer nothing of great interest. There is a significant negative correlation between age and BP performance, suggesting better performance by younger members of the group, and there is a correlation between IQ and recognition accuracy. The latter may be a statistical fluke, but one ad hoc explanation is that it reflects the low-frequency target words used in the recognition test. It is possible that some of the lower IQ younger subjects did not know what some of the target items meant and that this undermined their recognition performance.

In sum, the present data indicate that at least two factors must be invoked to explain age-related deficits in BP performance. Part of the deficit appears to be due to faulty acquisition storage, and part consists of a retrieval deficit linked to frontal dysfunction. Furthermore, both of these deficits appear constant as a function of retention inter$\mathrm{val}$, thus supporting the view that age-related deficits on the BP task do not arise from differential rates of forgetting. The present data also strengthen the view that a significant aspect of age-related memory loss arises through atrophy of the frontal lobes.

\section{REFERENCES}

BAddeley, A. D. (1987). Working memory. Oxford, U.K.: Oxford University Press.

Benton, A. L., Hamsher, K., Varney, N., \& Spreen, O. (1983). Contributions to neuropsychological assessment. New York: Oxford University Press.

BRoWN, J. (1959). Some tests of the decay theory of immediate memory. Quarterly Journal of Experimental Psychology, 10, 12-21.

Craik, F. I. M., Morris, L. W., Morris, R. G., \& Loewen, E. R. (1990). Relations between source amnesia and frontal lobe functions in older adults. Psychology \& Aging, 5, 148-151.

Gardiner, J. M., Craik, F. I. M., \& BirTwhistle, J. (1972). Retrieval cues and release from proactive inhibition. Journal of Verbal Learming \& Verbal Behavior, 11, 778-783.

GARDINER, J. M., PARKIN, A. J. (1990). Attention and recollective experience in recognition memory. Memory \& Cognition, 18, 579-583.

Gerard, G., \& WeISBerg, L. A. (1986). MRI periventricular lesions in adults. Neurology, 36, 998-1001.

HaAland, K. Y., Vranes, L. F., Goodwin, J. S., d Garry, P. J. (1987). Wisconsin card sort test performance in a healthy old population. Journal of Gerontology, 42, 345-346.

HaUG, H. (1983). Anatomical changes in aging brain. In J. CervosNavarro \& H. I. Sarkander (Eds.), Neuropharmacology (Vol. 21). New York: Raven Press.

Janowsky, J. S., Shimamura, A. P., \& SQuire, L. R. (1989). Memory and metamemory: Comparisons between patients with frontal lobe lesions and amnesic patients. Psychobiology, 17, 3-11.

KAUSLER, D. H. (1982). Experimental psychology and human aging. New York: Wiley.

Leng, N. R. C., \& PArkin, A. J. (1989). Aetiological variation in the amnesic syndrome: Comparisons using the Brown-Peterson task. Cortex, 25, 251-259.

MCENTEE, W. J., \& Crook, T. H. (1990). Age-associated memory impairment. Neurology, 40, 526-530.

MiLler, E. (1984). Verbal fluency as a function of a measure of verbal intelligence and in relation to different types of cerebral pathology. British Journal of Clinical Psychology, 23, 53-57.

MitTenberG, W., SeidenberG, M., OLeary, D. S., \& DiGullo, D. V. (1989). Changes in cerebral functioning associated with normal aging. Journal of Clinical \& Experimental Neuropsychology, 11, 918-932.

Moscovitch, M., WINOCUR, G. (1983). Contextual cues and release from proactive inhibition in young and old people. Canadian Journal of Psychology, 83, 331-344.

Nelson, H. E. (1976). A modified card sorting test sensitive to frontal lobe deficits. Cortex, 12, 313-324.

Nelson, T. H., O'Connell, A. (1978). Dementia: The estimation of premorbid intelligence levels using the New Adult Reading Test. Cortex, 14, 234-244.

Parkin, A. J., Bell, W. P., \& Leng, N. R. C. (1988). A study of metamemory in amnesic and normal subjects. Cortex, 24, 33-39.

Parkin, A. J., Hunkin, N. M., Rees, J. E. (1991). Selective memory impairment following left thalamic infarction. Manuscript in preparation.

Parkin, A. J., Leng, N. R., Stanhope, N., \& Smith, A. P. (1988). Memory impairment following ruptured aneurysm of the anterior communicating artery. Brain \& Cognition, 7, 231-243.

PARKIN, A. J., \& Walter, B. (1991). Recollective experience, ageing, and frontal lobe dysfunction. Manuscript submitted for publication.

Peterson, L. R., \& Peterson, M. J. (1959). Short term retention of individual verbal items. Journal of Experimental Psychology, 58, 193-198. 
Schacter, D. L., Harbluk, J. L., Mclachlan, D. R. (1984). Retrieval without recollection: An experimental analysis of source amnesia. Joumal of Verbal Leaming \& Verbal Behavior, 23, 593-611.

Shallice, T., Evans, M. E. (1978). The involvement of the temporal lobes in cognitive estimation. Cortex, 14, 294-303.

SQUiRE, L. R. (1982). Comparisons between forms of amnesia: Some deficits are unique to Korsakoff's syndrome. Journal of Experimental Psychology: Learning, Memory, \& Cognition, 8, 560-571.

Squire, L. R. (1987). Memory and brain. New York: Oxford University Press.

Stuss, D. T., Kaplan, E. F., Benson, D. F., Weir, W. S., Chiull, S., a Sarazin, F. F. (1982). Evidence for involvement of orbito-frontal cortex in memory functions: An interference effect. Journal of Comparative \& Physiological Psychology, 96, 913-925.

WEEKS, D. J. (1988). The anomalous sentences repetition test. Atlantic Highlands, NJ: National Foundation for Educational Research.

Winocur, G., Moscovitch, M., * Witherspoon, D. (1987). Contextual cuing and memory performance in brain-damaged amnesics and old people. Brain \& Cognition, 6, 129-141.
Winocur, G., Oxbury, S., Roberts, R., Agnetti, V., a Davis, C. (1984). Amnesia in a patient with bilateral lesions to the thalamus. Neuropsychologia, 22, 123-143.

\section{NOTES}

1. The term short-term memory is not meant to imply that this paradigm loads specifically on a putative short-term storage or primary memory system. Use of the term is purely descriptive, denoting the relatively short periods of time over which retention is tested.

2. The subjects were further required to classify the subjective experience associated with each of these recognition responses. These data are not relevant to the present study, but they can be found in Parkin and Walter (1991).

(Manuscript received November 26, 1990; revision accepted for publication February 13, 1991.)

\section{Forthcoming Psychobiology Articles}

The following is a list of forthcoming Psychobiology articles that are currently in press. They are given in approximate order of acceptance. Each entry includes the name and address of the author with whom to communicate for further prepublication information.

"Effects of the peripheral 5-HT2 antagonist xylamidine on consummatory behaviors"' by S. Edwards \& R. Stevens (S.E., AFRC Institute of Food Research, Shinfield, Reading, Berkshire RG2 9AT U.K.)

"Prenatal protein malnutrition impairs visual discrimination learning in adult rats" by J. Tonkiss, J.R. Galler, B. Shukitt-Hale, \& F.J. Rocco (J.T., Ctr. for Behv'l. Devel. \& Mental Retardation, M921, Boston Univ. School of Medicine, 80 E. Concord St., Boston, MA 02118)
"How does the rat (Rattus norvegicus) adjust food-carrying responses to the influences of distance, effort, predatory odor, food size and food availability?" by I.Q. Whishaw \& H.C. Dringenberg (I.Q.W., Dept. of Psychology, Univ. of Lethbridge, 4401 University Dr., Lethbridge, AB, Canada T1K 3M4)

"Evocation in paradoxical sleep of a hippocampal conditioned cellular response acquired during waking" by $\mathrm{C}$. Maho, E. Hennevin, B. Hars, \& S. Poincheval (E.H., LPN2, C.N.R.S., 91198 Gif-sur-Yvette Cedex, France) 